\title{
Peritoneal fluid modulates the sperm acrosomal exocytosis induced by $\mathrm{N}$ - acetylglucosaminyl neoglycoprotein
}

E.P. Passos ${ }^{1}$, L. Brugnara' ${ }^{1}$ A.C. Facin ${ }^{1}$, A. Riffel ${ }^{1}$,

G.R. Lima², V. Freitas² and A. Brandelli ${ }^{1}$
${ }^{1}$ Setor de Reprodução Assistida, Serviço de Ginecologia e Obstetrícia, Hospital de Clínicas de Porto Alegre, Porto Alegre, RS, Brasil

Escola Paulista de Medicina, U niversidade Federal de São Paulo, São Paulo, SP, Brasil

\section{Correspondence \\ E.P. Passos \\ Serviço de Ginecologia e \\ O bstetrícia, Hospital de \\ Clínicas de Porto Alegre \\ Rua Ramiro Barcellos, 2350 \\ 90035-003 Porto Alegre, RS \\ Brasil \\ Fax: +55-51-346-7155 \\ Research supported by \\ HCPA and CNPq. Publication \\ supported by FAPESP.}

Received March 4, 1998

Accepted October 9, 1998

\begin{abstract}
The effect of peritoneal fluid (PF) on the human sperm acrosome reaction (AR) was tested. Sperm was pre-incubated with PF and the AR was induced by calcium ionophore A23187 and a neoglycoprotein bearing $\mathrm{N}$-acetylglycosamine residues (NGP). The AR induced by calcium ionophore was inhibited $40 \%$ by $\mathrm{PF}$ from controls $(\mathrm{PFc})$ and $50 \%$ by $\mathrm{PF}$ from the endometriosis (PFe) group, but not by $\mathrm{PF}$ from infertile patients without endometriosis (PFi). No significant differences were found in the spontaneous AR. When the AR was induced by NGP, pre-incubation with PFc reduced $(60 \%)$ the percentage of $A R$, while PFe and PFi caused no significant differences. The average rates of acrosome reactions obtained in control, NGP- and ionophoretreated sperm showed that NGP-induced exocytosis differed significantly between the PFc (11\%) and PFe/PFi groups (17\%), and the ionophore-induced AR was higher for PFi (33\%) than $\mathrm{PFc} / \mathrm{PFe}(25 \%)$. The incidence of the NGP-induced AR was reduced in the first hour of pre-incubation with PFc and remained nearly constant throughout $4 \mathrm{~h}$ of incubation. The present data indicate that $\mathrm{PF}$ possesses a protective factor which prevents premature AR.
\end{abstract}

\section{Introduction}

Mammalian sperm must undergo a specialized exocytotic event, the acrosome reaction (AR), to fertilize the oocyte. The binding of capacitated sperm to the zona pellucida triggers the physiological AR, whose proposed mechanism occurs by aggregation of specific receptors on the sperm surface $(1,2)$. Sperm-egg binding involves lectinlike proteins on the sperm surface that recognize specific glycans of the zona pellucida (3), and multiple carbohydrates appear to be involved in human sperm-zona pellucida

\section{Key words}

- Acrosome reaction

- Endometriosis

- Infertility

- Peritoneal fluid

- Sperm binding (4). Binding sites for mannose and $\mathrm{N}$-acetylglucosamine have been proposed to play an important role in human fertilization. They are involved in the induction of AR by $\mathrm{N}$-acetylglucosamine- and mannose-containing neoglycoproteins (NGP) (5-7) and are related to the fertilizing potential of spermatozoa (8-10). Moreover, the stimulation of human sperm AR by neoglycoproteins is strongly correlated with the rate of fertilization in vitro $(9,10)$, and may be used to predict fertilization success.

Peritoneal fluid (PF) may be introduced into the tubal enviroment, where its soluble 
substances or cells interact with the gametes and may act as modulatory factors in fertilization. Some components of PF are elevated in or unique to endometriosis patients $(11,12)$. The composition of PF may be altered in women with endometriosis or unexplained infertility, and these substances may have a detrimental effect on sperm and/or oocyte. The effects of PF from women with endometriosis on some sperm functional parameters have been reported by several investigators, resulting in conflicting observations (11-15). Moreover, peritoneal fluid from patients with endometriosis decreases the sperm-egg interaction in mice (16) and spermzona binding in humans (17). The effects of PF on sperm function are not clear and require further studies.

The aim of the present study was to determine the effect of PF from fertile women and from infertile patients with and without endometriosis on the sperm acrosome reaction induced by an $\mathrm{N}$-acetylglucosamine neoglycoprotein.

\section{Material and Methods}

\section{Reagents}

Calcium ionophore A23187 (Sigma Chemical Co., St. Louis, MO) was dissolved as a $100 \mathrm{x}$ stock solution in dimethyl sulfoxide and stored at $-20^{\circ} \mathrm{C}$. GPM medium was obtained from Serono (Madrid, Spain). Bovine serum albumin (BSA; fatty acid and globulin free) and N-acetyl-ß-D-glucosaminide-phenylisothiocyanate were from Sigma. All other reagents were from Merck (Darmstadt, Germany) unless otherwise stated.

\section{Synthesis of neoglycoproteins}

The neoglycoprotein albumin N-acetylB-D-glucosaminide phenylisothiocyanate (BSA-GlcNAc) was obtained essentially as described by Monsigny et al. (18). Briefly, to a solution of $1 \mathrm{mmol}$ of BSA in $5 \mathrm{ml} 0.1 \mathrm{M}$ sodium carbonate buffer, $\mathrm{pH}$ 9.5, $20 \mathrm{mmol}$ of N-acetyl-ß-D-glucosaminide phenylisothiocyanate was added. The solution was stirred for $20 \mathrm{~h}$ at $4^{\circ} \mathrm{C}$, and the product was then purified by gel filtration on a Sephadex G-50 column in distilled water. The carbohydrate content, determined by the phenolsulfuric acid method (19), was 23 residues per BSA molecule. The neoglycoprotein BSA-GlcNAc was dissolved in phosphate buffered saline (PBS) at $100 \mathrm{mg} / \mathrm{ml}$ for stock solutions, and stored at $-20^{\circ} \mathrm{C}$ in small aliquots that were thawed before use.

\section{Peritoneal fluid}

Peritoneal fluid was obtained from 15 women of reproductive age undergoing a diagnostic laparoscopy or seeking tubal ligation. The sample was separated and pooled into three groups: fertile without endometriosis $(\mathrm{PFc} ; \mathrm{N}=5)$; infertile without endometriosis $(\mathrm{PFi} ; \mathrm{N}=5)$, and infertile with endometriosis ( $\mathrm{PFe} ; \mathrm{N}=5)$. Peritoneal fluid was collected by laparoscopy, placed on ice and immediately transported to the laboratory in sterile plastic tubes. After centrifugation (10 $\mathrm{min} / 200 \mathrm{~g}$ at $4^{\circ} \mathrm{C}$ ) the supernatant was aliquoted and frozen at $-70^{\circ} \mathrm{C}$ until use. All women were ovulating and none was using any contraceptive method. Procedures were carried out between days 7-10 of the menstrual cycle. The PFs from patients with endometriosis used in this study correspond to stages 1 and 2 according to the classification of the American Fertility Society (20). Protein concentration was determined by the method of Lowry et al. (21): $\mathrm{PFc}=22.6 \pm 3.5$ $\mathrm{mg} / \mathrm{ml} ; \mathrm{PFe}=24.7 \pm 5.6 \mathrm{mg} / \mathrm{ml} ; \mathrm{PFi}=23.8 \pm$ $3.5 \mathrm{mg} / \mathrm{ml}$.

\section{Sperm capacitation}

Semen samples were obtained from 12 donors in our assisted reproduction program, presenting normal sperm morphology and 
progressive motility. Only samples with more than $80 \%$ motile cells were used. After complete liquefaction, semen was diluted in the same volume of GPM medium, and then centrifuged for $10 \mathrm{~min}$ at $900 \mathrm{~g}$. The supernatant was discarded and the sperm was washed twice in GPM. Motile sperm were obtained by swim-up, diluted to $5 \times 10^{6}$ cells/ $\mathrm{ml} \mathrm{GPM}$ and incubated for $18 \mathrm{~h}$ at $37^{\circ} \mathrm{C}$ and $5 \% \mathrm{CO}_{2}$ in air.

\section{Acrosome reaction assays}

Fifty microliters of GPM (for mock incubations), $\mathrm{PFc}, \mathrm{PFe}$ or PFi were added to 450 $\mu l$ of capacitated sperm suspensions and incubated for $60 \mathrm{~min}$. Aliquots $(100 \mu \mathrm{l})$ of PFincubated sperm were then separately exposed for $60 \mathrm{~min}$ to the following reagents at the indicated final concentration: BSAGlcNAc $(2 \mu \mathrm{g} / \mathrm{ml})$, calcium ionophore A23187 $(10 \mu \mathrm{M})$ (positive control), or fresh medium (negative control). Induction of AR was stopped by adding $1 \mathrm{ml}$ of PBS, and washing cells twice with PBS. An aliquot of sperm suspensions was air dried onto immunofluorescence slides, submerged $30 \mathrm{~s}$ in methanol at $4^{\circ} \mathrm{C}$ for cell permeabilization (22) and dried again immediately. The acrosome reaction was evaluated using lectin staining with $50 \mu \mathrm{g} / \mathrm{ml}$ FITC-labeled Pisum sativum agglutinin in duplicate assays. Stained cells (approximately 200 cells per treatment) were scored under an epifluorescence microscope at $1000 \mathrm{X}$ magnification. The stimulation of the AR by neoglycoprotein (STIM) was calculated as:

$$
\mathrm{STIM}=\frac{\% \mathrm{ARn}-\% \mathrm{ARc}}{\% \mathrm{ARi}-\% \mathrm{ARc}}
$$

where $\% \mathrm{ARn}=$ percentage of acrosome reaction in NGP-treated cells; \%ARc = percentage of acrosome reaction in cells incubated with medium, and \%ARi = percentage of acrosome reaction in ionophore-treated cells.

\section{Statistical analysis}

The means \pm SEM were calculated for the variables studied. The data were subjected to analysis of variance and, when appropriate, to Duncan analysis to determine differences. Values were considered significantly different from each other when $\mathrm{P}<0.05$.

This research was approved by the Ethics Committee of the hospital (GPPG/HCPA, register No. 95051) and informed consent was obtained from all subjects involved in the study.

\section{Results}

Human sperm acrosomal exocytosis was induced with NGP and calcium ionophore after pre-incubation with peritoneal fluid from infertile women with (PFe) and without endometriosis (PFi). Peritoneal fluid from fertile women $(\mathrm{PFc})$ was used as control. The results are summarized in Figure 1. In the absence of inducer (spontaneous AR), pre-incubation with the different PF groups caused no significant effect on the levels of acrosomal loss. The induction of AR by calcium ionophore A23187 was used as a full response control. The ionophore-induced AR was inhibited when sperm was pre-treated with $\mathrm{PFc}$ or $\mathrm{PFe}$, while treatment with $\mathrm{PFi}$

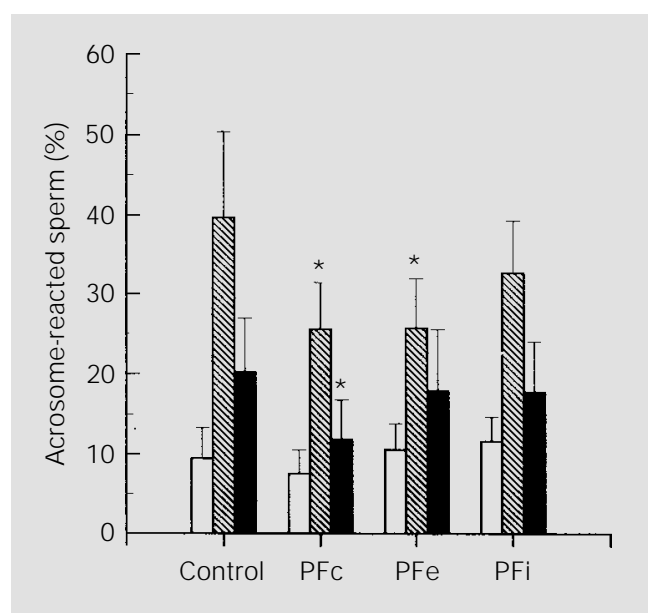

Figure 1 - Effect of peritoneal fluid (PF) on the sperm acrosome reaction. Cells were preincubated with PFc (PF from fertile women, control), PFe (PF from patients with endometriosis), PFi (PF from infertile women) or GPM (control, no PF was added), and then incubated with $10 \mu \mathrm{M}$ calcium ionophore A23187 (dashed bars), $2 \mu \mathrm{g} / \mathrm{ml}$ GlcNAc-neoglycoprotein (black bars) or GPM medium (grey bars). Results are reported as the mean \pm SEM of 12 sperm samples. ${ }^{*} \mathrm{P}<0.05$ vs control group (Duncan test). 
Figure 2 - Effect of peritoneal fluid (PF) on the sperm acrosome reaction induced by neoglycoprotein. Sperm were incubated with PF from fertile women (PFC) or GPM medium as control (C). Data are reported as acrosome-reacted sperm (\%) for $\mathrm{N}=12$ samples in each group.
Figure 3 - Frequency of the NGPinduced acrosome reaction during incubation of sperm with PFc, PFe or PFi. Values for PFC were significantly different from controls at all time points after 30 min. In contrast, values for $\mathrm{PFe}$ and PFi were not different from controls at any time. Data are reported as the mean \pm SEM of three replicate experiments. caused no significant difference (Figure 1). The effect of both PFc or PFe on the ionophore-induced AR was similar, presenting no statistical difference. When acrosomal exocytosis was induced with NGP, only the treatment with $\mathrm{PFc}$ resulted in a significant decrease in the incidence of AR (Figure 1), while pre-incubation with PFe or PFi caused no significant effects.

$\mathrm{PFc}$ data for individuals are shown in Figure 2. Among the samples studied only two presented a slight increase in AR rate when treated with $P F c$, while in the others pre-incubation with PFc inhibited the AR. Some patients showed a low incidence of
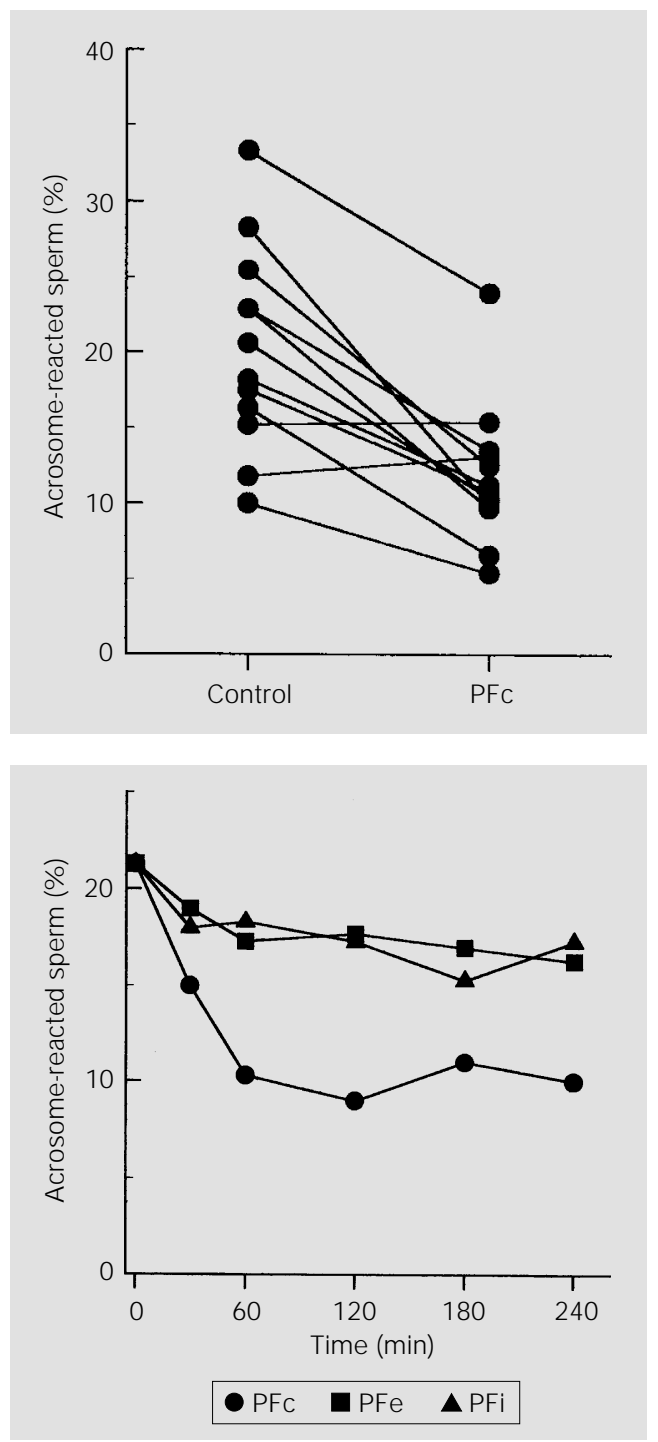

NGP-induced acrosomal loss. The large SEM observed indicate wide variations in individual responses, but, as can be seen in Figure 2, wide interindividual variations in AR also exist.

The kinetics of the PF effect on the NGPinduced AR are shown in Figure 3. The incidence of NGP-induced AR among spermatozoa was reduced in the first hour of preincubation with PFc and remained nearly constant for the next $4 \mathrm{~h}$ of incubation. In contrast, the addition of PFe or PFi did not significantly affect the rate of AR induced by NGP at any time point examined (Figure 3 ).

The normalized NGP-induced AR, represented by the value of STIM, presented a positive correlation with the rate of in vitro fertilization (9), being a functional test to predict the fertilization success. Adequate in vitro fertilization rates are obtained only when the STIM values are above or near 0.2 , which is considered to be the cut-off value (9). The STIM values were calculated for the PFs tested, and all groups presented STIM $>0.2$ (Figure 4). Although PFc values were lower than for other treatments, there were no statistical differences between groups.

\section{Discussion}

The substances or cells present in the PF may be deleterious or, conversely, may enhance some sperm functional parameters. Since the composition of PF from infertile women with or without endometriosis is altered, its effect on sperm function may be modified. In this regard, activated macrophages that phagocytize sperm cells (12) and activated lymphocytes and their products are modified in the PF from women with endometriosis $(14,23)$. Moreover, some PF constituents are elevated or unique to endometriosis patients $(24,25)$, but have not been studied in terms of their effect on sperm function.

The physiological induction of the acrosome reaction by the zona pellucida in ca- 
pacitated sperm requires the normal function of at least two mechanisms. First, the complementary binding of sperm-surface receptors to zona pellucida glycoproteins, and secondly, the transduction of this signal with activation of calcium flux. Neoglycoproteins containing mannose or N-acetylglucosamine residues induce the human sperm AR, with requirements similar to those of the zona pellucida (5). The NGP effect occurs by signal transduction pathways similar to those described for zona-induced AR, i.e., activation of G-proteins, voltage-dependent calcium channels (26) and tyrosine kinase (6). The induction of AR by GlcNAcneoglycoprotein also presents a positive correlation with in vitro fertilization rates (9). In contrast, calcium ionophore triggers the AR independently of the capacitation process and without the involvement of membrane receptors. The lack of a relationship between the results of chemical (ionophore) and physiological (zona pellucida) stimuli for the acrosome reaction has been recently reported (27). In view of the physiological significance of NGP-induced AR, the results presented here suggest that PF from fertile women contains a protective factor which preserves the sperm against a premature AR. This indicates an inhibitory effect on sperm AR which was not observed with PFe and PFi. In addition, the STIM values obtained were similar for all three groups of PF studied, being above 0.2 , which is the value related to successful in vitro fertilization. This suggests the effect of PFc in protecting against a premature AR, but without impairing fertilization.

The effect of PF on sperm AR has been reported by others. The incubation of sperm with PF from patients with endometriosis or unexplained infertility increases the spontaneous or dibutyryl c-AMP-induced AR (15) when compared with untreated cells. However, the effect of PF from fertile women was not assessed for comparison. Arumugam (28) reported that elevated iron concentrations in

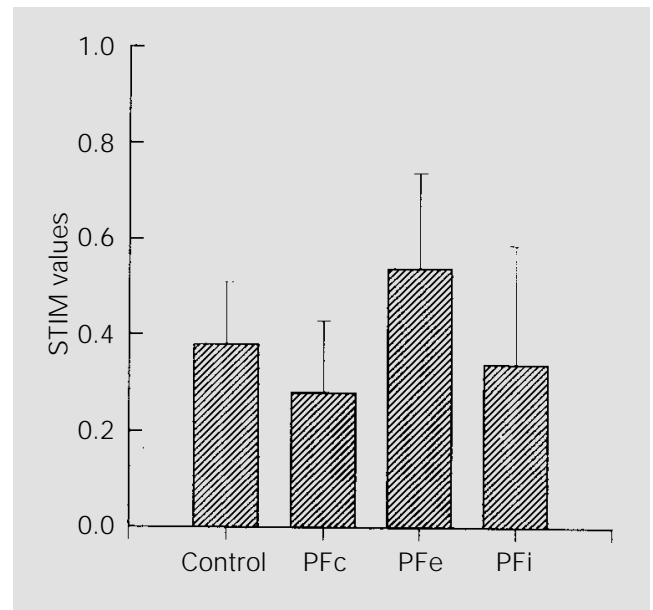

Figure 4 - Stimulation of acrosome reaction in response to neoglycoprotein. The STIM values were calculated as described in Material and Methods. Results are reported as the mean \pm SEM of 12 sperm samples. There were no stastistical differences. the PF from endometriosis had a detrimental effect on the AR. The inhibitory effect of PF from endometriosis patients on both spontaneous and ionophore-induced AR in comparison with spermatozoa incubated in $\mathrm{PF}$ from the control group has been recently reported (29). Finally, our findings demonstrate that PFc inhibits the AR induced by NGP and that PF from control and endometriosis groups caused a decrease in the ionophore-induced AR. These effects of PF on acrosome reaction may be explained in part by the complex composition of each sample of PF. Furthermore, some differences among procedures, such as the concentration of PF used, may contribute to the contradictory reports from different laboratories.

The PF in cases of endometriosis-associated infertility may produce adverse effects on the normal mechanisms of gamete interaction. Sueldo et al. (16) reported that PFe decreases sperm-egg adhesion in the mouse. The reduction of sperm binding to the zona pellucida in the presence of PF from patients with endometriosis has been reported, but it is not known whether changes were occurring at the sperm or oocyte level (17). Factors like endometrial protein PP14, that do not affect the in vitro AR, may decrease the in vivo $\mathrm{AR}$ by inhibiting the sperm-zona binding (30). Indeed, PF from infertile women presented elevated glycosidase activities, which may promote adverse effects on fer- 
tilization (31). Since PF should be present at the fertilization site, and human sperm must be unreacted to bind to the zona pellucida, our data suggest that sperm may undergo an ealier AR in contact with the PF from infertile women.

\section{Acknowledgments}

The authors thank the Setor de Imunologia, HCPA, for microscopy support.

\section{References}

1. Leyton L \& Saling PM (1989). Evidence that aggregation of sperm receptors by ZP3 triggers the acrosome reaction. J ournal of Cell Biology, 108: 2163-2168.

2. Macek MB, Lopez LC \& Shur BD (1991). Aggregation of $ß-1,4$-galactosyltransferase on mouse sperm induces the acrosome reaction. Developmental Biology, 147: 440-444.

3. Macek MB \& Shur BD (1988). Proteincarbohydrate complementarity in mammalian gamete recognition. Gamete Research, 20: 93-109.

4. Miranda PV, Echeverria FG, Briggiler CIM, Brandelli A, Blaquier JA \& Tezon JG (1997). Glycosidic residues involved in human sperm-zona pellucida binding in vitro. Molecular Human Reproduction, 3: 309-404.

5. Brandelli A, Miranda PV \& Tezon JG (1994). Participation of glycosylated residues in the human sperm acrosome reaction. Possible role of $\mathrm{N}$-acetylglucosaminidase. Biochimica et Biophysica Acta, 1220: 299-304.

6. Benoff S, Hurley IR, Mandel FS, Barcia M, Cooper GW \& Hershlag A (1995). Use of mannose ligands in IVF screens to mimic zona pellucida-induced acrosome reactions and phosphotyrosine kinase activation. Fertility and Sterility, 63 (Suppl): S38S39.

7. Benoff S, Hurley IR, Mandel FS, Cooper GW \& Hershlag A (1997). Induction of the human sperm acrosome reaction with mannose-containing neoglycoprotein ligands. Molecular Human Reproduction, 3: 827-837.

8. Benoff S, Cooper G, Hurley I, Napolitano B, Rosenfeld DL, Scholl GM \& Hershlag A (1993). Human sperm fertilizing potential in vitro is correlated with differential expression of a head-specific mannoseligand receptor. Fertility and Sterility, 59: 854-862.

9. Brandelli $A$, Miranda PV, Añon-Vazquez MG, Briggiler CIM, Sanjurjo C, Echeverria FG, Blaquier JA \& Tezon J G (1995). A new predictive test for in vitro fertilization based on the induction of sperm acrosome reaction by $\mathrm{N}$-acetylglucosamineneoglycoprotein. Human Reproduction, 10: 1751-1756.

10. Benoff S, Hurley IR, Mandel FS, Paine T, J acob A, Cooper GW \& Hershlag A (1997). Use of mannose ligands in IVF screens to mimic zona pellucida-induced acrosome reactions and predict fertilization success. Molecular Human Reproduction, 3: 839846.

11. Chacho KJ, Chacho MS, Andersen PJ \& Scommegna A (1986). Peritoneal fluid in patients with and without endometriosis: prostanoids and macrophages and their effect on the sperm penetration assay. American J ournal of Obstetrics and Gynecology, 154: 1290-1299.

12. Hill J A, Haimovici F, Politch J A \& Anderson DJ (1987). Effects of soluble products of activated lymphocytes and macrophages (lymphokines and monokines) on human sperm motion parameters. Fertility and Sterility, 47: 460-465.

13. Halme J \& Hall J L (1982). Effect of pelvic fluid from endometriosis patients on human sperm penetration of zona-free hamster ova. Fertility and Sterility, 37: 573576.

14. Burke RK (1987). Effect of peritoneal fluid washings from women with endometriosis on sperm velocity. J ournal of Reproductive Medicine, 10: 743-745.

15. Bielfeld P, Graf MA, J eyendran RS, De Leon FD \& Zaneveld LJ D (1993). Effects of peritoneal fluid from patients with endometriosis on capacitated sperm. Fertility and Sterility, 60: 893-896.

16. Sueldo $C E$, Lambert $H$, Steinleitner $A$, Rathwick G \& Swanson J (1987). The effect of peritoneal fluid from patients with endometriosis on murine sperm-oocyte interaction. Fertility and Sterility, 48: 697699.

17. Coddington $\mathrm{CC}$, Oehninger $\mathrm{S}$, Cunninghan DS, Hansen K, Sueldo CE \& Hodgen GD (1992). Peritoneal fluid from patients with endometriosis decreases sperm binding to the zona pellucida in the hemizona assay: a preliminary report. Fertility and Sterility, 57: 783-786.

18. Monsigny $M$, Roche $A C \&$ Midoux $P$ (1984). Uptake of neoglycoproteins via membrane lectin(s) of L1210 cells evidenced by quantitative flow cytofluorometry and drug targeting. Biology of the Cell, 51: 187-196.

19. Chaplin MF (1986). Monosaccharides. In: Chaplin MF \& Kennedy J F (Editors), Carbohydrate Analysis. IRL Press, Oxford, 136.

20. American Fertility Society (1985). Classification of endometriosis. Fertility and Sterility, 43: 351-352.

21. Lowry OH, Rosembrough NJ, Farr AL \& Randall RJ (1951). Protein measurement with the Folin phenol reagent. J ournal of Biological Chemisty, 193: 267-275.

22. Mendoza C, Carreras A, Moos J \& Tesarik J (1992). Distinction between true acrosome reaction and degenerative acrosome loss by a one-step staining method using Pisum sativum agglutinin. J ournal of Reproduction and Fertility, 95: 755-763.

23. Ho HN, Chao KH, Chen HF, Wu MY, Yang YS \& Lee TY (1995). Peritoneal natural killer cytotoxicity and CD25+CD3+ lymphocyte subpopulation are decreased in women with stage III-IV endometriosis. Human Reproduction, 10: 2671-2675.

24. Koninckx PR, Riittinen L, Seppala M \& Cornillie FJ (1992). CA-125 and placental protein 14 concentrations in plasma and peritoneal fluid of women with deeply infiltrating pelvic endometriosis. Fertility and Sterility, 57: 523-530.

25. Nothnick WB, Curry J r TE, Muse KN, London SN \& Vernon MW (1994). Detection of a unique $32-\mathrm{kd}$ protein in the peritoneal fluid of women with endometriosis. Fertility and Sterility, 61: 288-293.

26. Brandelli A, Miranda PV \& Tezon J G (1996). Voltage-dependent calcium channels and $\mathrm{Gi}$ regulatory protein mediate the human sperm acrosomal exocytosis 
induced by $\mathrm{N}$-acetylglucosaminyl/mannosyl neoglycoproteins. J ournal of Andrology, 17: 522-529.

27. Liu DY \& Baker HWG (1996). A simple method for assessment of the human acrosome reaction of spermatozoa bound to the zona pellucida: lack of relationship with ionophore A23187-induced acrosome reaction. Human Reproduction, 11: 551-557.

28. Arumugam K (1994). Endometriosis and infertility: raised iron concentration in the peritoneal fluid and its effect on the acrosome reaction. Human Reproduction, 9: 1153-1157.

29. Tasdemir M, Tasdemir I, Kodama H \& Tanaka T (1995). Effect of peritoneal fluid from infertile women with endometriosis on ionophore-stimulated acrosome loss. Human Reproduction, 10: 24192422.

30. Oehninger S, Coddington CC \& Seppala
M (1993). Endometrial protein PP14 reduces the sperm-zona pellucida binding. A mechanism for endometriosis-related infertility. Conjoint Meeting of the American Fertility Sterility Society and the Canadian Fertility and Andrology Society, October 11-14, Montreal, Quebec, S19.

31. Brandelli A \& Passos EP (1998). Glycosidases in the peritoneal fluid from infertile women with and without endometriosis. Clinical Biochemistry, 31: 181-186. 